with fall prevalence and frequency, respectively. Adjustments were made for sex, height, BMI, socioeconomic position, physical activity, smoking, knee pain, diabetes, cardiovascular events and respiratory and depressive symptoms.

Results Women reported higher prevalence of falls than men at ages $60-64(23 \%$ vs $14 \%)$ and 68 (26\% vs $18 \%)$. Longer balance time at age 53 was associated with reduced odds of falling at age 60-64 [OR: 0.98 (95\% CI: 0.97,1.00)]; similar associations were found between balance at age 60-64 and falls at age $68[0.96(0.93,0.99)]$. Better balance at age 53 was associated with lower risk of 3 or more falls (vs no falls) at ages 60-64 [RRR: $0.88 \quad(0.80,0.98)]$ and $68 \quad[0.93$ $(0.88,0.97)]$. Better balance at age $60-64$ was also associated with lower risk of $3+$ falls at age 68 [RRR: $0.92(0.85,0.98)$ ] and in addition was associated with lower risk of 1-2 falls [0.97 (0.94,1.00)]. These associations remained after adjustments.

Discussion Poorer balance at ages 53 and 60-64 was associated with subsequent fall risk. Balance at age 53 was most strongly associated with 3 or more falls, while balance at age 60-64 was associated with both $1-2$ and $3+$ falls. Whether this is due to stronger associations at age 60-64 or a shorter time between balance and falls assessments requires further investigation. Balance tests in middle age may help identify high risk individuals who would benefit from earlier interventions to prevent future recurrent falls.

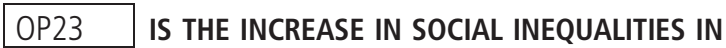 INFLAMMATION WITH AGE UNDERESTIMATED IN CONVENTIONAL LONGITUDINAL ANALYSES? SOCIOECONOMIC POSITION AND REPEATED SYSTEMIC INFLAMMATION IN OLDER ADULTS LIVING IN ENGLAND}

G Chatzi*, T Chandola, A Cernat, N Shlomo. Cathie Marsh Institute for Social Research, Social Statistics, University of Manchester, Manchester, UK

\subsection{6/jech-2019-SSMabstracts.23}

Background Although social inequalities in health are consistently observed across the lifecourse in different populations, there are methodological issues in describing whether these inequalities decrease in later life. The association between socioeconomic adversity and systemic inflammation is well documented in cross-sectional studies, however, the association between living in socioeconomic disadvantage and repeated systemic inflammation in older adults has not been examined in detail, particularly taking into account longitudinal patterns of missingness. Inference from longitudinal analyses of ageing populations is susceptible to biases arising from attrition and non-random dropout. The accumulation of drop-outs over several waves reduce the representativeness of the study population and certain subpopulations can be over or underrepresented in the sample.

Methods 4,574 men and women aged 52 years and older from the English Longitudinal Study of Ageing (ELSA) wave 2 onwards were analysed. ELSA is a prospective cohort study that is representative of the English population. C - reactive protein levels were measured in waves 2, 4, 6, and 8 (20042016). Latent growth curve models estimated the relationship between different measures of socioeconomic position (education, wealth, and social class) and C - reactive protein, compensating for missing data under different assumptions: complete case analysis, full information maximum likelihood, multiple imputation, Diggle-Kenward selection model, and pattern-mixture model. All models were adjusted for gender, age, ethnicity, and marital status.

Results At baseline in wave 2, we found differences between the most and least affluent categories of socioeconomic position. Participants with foreign or no qualifications $(0.16 \mathrm{log}$ $(\mathrm{mg} / \mathrm{l}), 95 \%$ CI $0.09-0.23)$, participants in the lowest wealth tertile $(0.24 \log (\mathrm{mg} / \mathrm{l}), 95 \%$ CI $0.16-0.32)$, and participants in manual occupations $(0.13 \log (\mathrm{mg} / \mathrm{l}), 95 \%$ CI $0.07-0.19)$ had increased levels of C-Reactive protein compared to the most advantaged categories of education, wealth, and social class. Although, C - reactive protein levels decreased in later waves, the differences between the most and least socioeconomic advantaged groups remained large. Furthermore, differences between the Diggle-Kenward and other methods for compensating for missing data suggest that the missing completely at random and missing at random analyses underestimated socioeconomic differences in C-Reactive protein.

Conclusion This study demonstrates that living in socioeconomic disadvantage is associated with higher $\mathrm{C}$ - reactive protein levels over time and that the social discrepancies in health between the most and least affluent socioeconomic groups persist at older ages. It also highlights the importance of compensating for missingness in longitudinal studies with ageing participants who are susceptible to non-random drop out.

\section{OP24 SOCIAL AND CULTURAL ENGAGEMENT AND DEMENTIA INCIDENCE: COMPARISONS OF DIFFERENT TIME-TO- EVENT ANALYSES USING THE ENGLISH LONGITUDINAL STUDY OF AGEING}

D Fancourt* , A Steptoe, D Cadar. Department of Behavioural Science and Health, University College London, London, UK

\subsection{6/jech-2019-SSMabstracts.24}

Background There is a growing literature drawing on theories of cognitive reserve suggesting that factors relating to social networks, activity and support can predict dementia in older age. Much of this work has focused on social deficits (such as loneliness and isolation), but there is increasing evidence that engaging in social activities can be protective. The aim of this study was to compare the potential protective associations of between three different types of social activity (socialising, community group activities and cultural engagement) and dementia incidence.

Methods We used nationally-representative data from 9,550 adults aged $50+$ from the English Longitudinal Study of Ageing followed-up over a 12 year period. We determined dementia occurrence using an algorithm that combined self- or informant-reported physician diagnosis of dementia with informant-reported score on the Informant Questionnaire on Cognitive Decline in the Elderly (IQCODE). We ran three types of time-to-event analyses: Cox proportional hazards models (modelling time to dementia), competing risk regressions models (modelling time to dementia vs the competing risk of death), and modified Fine and Gray Subdistribution hazards models (modelling time to dementia or death with a high probability of dementia vs the competing risk of death with a low probability of dementia). Analyses controlled for all identified demographic, health-related, and social covariates. 
Results Social and cultural engagement were both independently associated with a lower risk of developing dementia in older age in fully-adjusted models. Using time-to-event analyses, socialising once a week was associated with a $29 \%$ lower risk $\mathrm{OR}=0.71 \quad 95 \% \mathrm{CI} \quad 0.52-0.97$ and cultural engagement every few months or more was associated with a $42 \%$ lower risk $\mathrm{OR}=0.5895 \% \mathrm{CI}$ 0.41-0.80. Using competing risk models, socialising $\mathrm{OR}=0.80 \quad 95 \% \mathrm{CI} \quad 0.59-1.08$ and cultural engagement $\mathrm{OR}=0.65$ 95\%CI $0.47-0.90$. Using modified Fine and Gray Subdistribution hazards models, socialising $\mathrm{OR}=0.66$ 95\%CI $0.53-0.82$ and cultural engagement $\mathrm{OR}=0.4295 \% \mathrm{CI}$ $0.32-0.56$. Community group activities were only associated with dementia in minimally-adjusted models. Results were robust to sensitivity analyses considering moderators, reverse causality, over-adjustment, and baseline cognitive function.

Conclusion The results presented here suggest that social and cultural engagement are independent risk-reducing factor for the development of dementia in older age. Even for those who lack contact with friends and family or who socialise infrequently, engagement with cultural venues, even on a less frequent basis, could be protective against the incidence of dementia. These findings align with broader findings relating to cognitive reserve and support the development of multimodal community-based interventions to promote healthy cognitive ageing amongst older adults.

\section{OP25 THE ASSOCIATION OF POOR ORAL HEALTH WITH A RANGE OF INFLAMMATORY MARKERS: RESULTS FROM TWO POPULATION BASED STUDIES OF OLDER PEOPLE IN THE UK AND USA}

\begin{abstract}
${ }^{1} \mathrm{E}$ Kotronia*, ${ }^{2} \mathrm{GS}$ Wannamethee, ${ }^{2} \mathrm{OA}$ Papacosta, ${ }^{3} \mathrm{PH}$ Whincup, ${ }^{2} \mathrm{LT}$ Lennon, ${ }^{4} \mathrm{RJ}$ Weyant, ${ }^{5}$ TB Harris, ${ }^{1,2}$ SE Ramsay. 'Institute of Health and Society, Newcastle University, Newcastle Upon Tyne, UK; ${ }^{2}$ Department of Primary Care and Population Health, UCL, London, UK; ${ }^{3}$ Population Health Research Institute, St George's University of London, London, UK; ${ }^{4}$ Department of Dental Public Health, School of Dental Medicine, University of Pittsburgh, Pittsburgh, USA; ${ }^{5}$ Laboratory of Epidemiology and Population Sciences, National Institute of Aging, Bethesda, USA
\end{abstract}

\subsection{6/jech-2019-SSMabstracts.25}

Background Oral health conditions such as tooth loss, periodontal (gum) disease and dryness of mouth are very common health problems in older people, with significant impacts on nutrition, quality of life and well-being of ageing populations. Oral health, particularly periodontal disease, has been linked to systemic inflammation, such as high C-reactive protein (CRP) and interleukin-6 (IL-6). However, very few studies of older people have investigated the associations between oral health and inflammation. We examined whether a range of oral health markers are associated with inflammatory markers in two population-based studies of older people in the UK and USA.

Methods Cross-sectional analyses were conducted in the British Regional Heart Study (BRHS) comprising men aged 71-92 $(n=2147)$ from 24 British towns, and the US Health, Aging and Body Composition (HABC) Study comprising men and women aged 71-80 $(n=3075)$. Assessments included oral health (periodontal disease, tooth count, dry mouth) and inflammatory markers such as CRP, IL-6, leptin, tissue plasminogen activator (tPA), von Willebrand Factor (vWF), fibrin Ddimer, high sensitivity Troponin $\mathrm{T}$ (hsTnT), N-Terminal prohormone of brain natriuretic peptide (NTproBNP), and proinsulin.
Results In the BRHS, having no natural teeth was associated with being in the top tertiles of CRP, and fibrin D-dimer (odds ratio $(\mathrm{OR})=1.35,95 \% \mathrm{CI}$ : $1.01-1.80 ; \mathrm{OR}=1.38,95 \%$ CI: 1.03-1.85, respectively) after adjustment for age, social class, smoking, history of cardiovascular disease and diabetes, and BMI. Tooth loss ( $<21$ teeth) was associated with being in the top tertiles of CRP, hsTnT, fibrin D-dimer, and NTproBNP (fully adjusted $\mathrm{OR}=1.31$, 95\%CI: 1.02-1.68; $\mathrm{OR}=1.32$, 95\%CI: $1.01-1.74 ; \mathrm{OR}=1.37$, 95\%CI: $1.05-1.77$, $\mathrm{OR}=1.40$, 95\% CI: 1.01-1.94, respectively). Periodontal disease was associated with being in the top tertile of hsTnT. In the HABC Study, having no teeth and partial tooth loss were associated with being in the top tertile of CRP $(\mathrm{OR}=1.57$, 95\% CI: 1.10-2.25; $\mathrm{OR}=1.40,95 \% \mathrm{CI}:$ 1.13-1.75, respectively) after adjustment for age, gender, race, education, smoking, history of cardiovascular disease and diabetes, and BMI. Moreover, having $\geq 3$ oral health problems was associated with being in the top tertiles of CRP and IL-6 after full adjustment.

Conclusion Poor oral health in older people was associated with increased levels of various inflammatory markers including CRP, fibrin D-dimer, hsTnT and NTproBNP. These findings suggest that poor oral health in older age is linked not only to general systemic inflammation but also inflammation associated with metabolic disturbances. These associations could offer insights into mechanistic pathways by which poor oral health could influence age-related conditions.

\section{Mental Health 1}

\section{OP26 PATIENT EXPERIENCE OF ENGAGEMENT WITH HEALTHCARE SERVICES FOLLOWING AN EPISODE OF HIGH RISK SELF-HARM: A MIXED METHODS STUDY}

\footnotetext{
${ }^{1,2} \mathrm{G}$ Cully, ${ }^{1,2} \mathrm{D}$ Leahy, ${ }^{1,3} \mathrm{~F}$ Shiely, ${ }^{1,2} \mathrm{E}$ Arensman*. 'School of Public Health, University College Cork, Cork, Ireland; ${ }^{2}$ National Suicide Research Foundation, Cork, Ireland; ${ }^{3}$ HRB Clinical Research Facility, Mercy University Hospital, Cork, Ireland
}

\subsection{6/jech-2019-SSMabstracts.26}

Background Hospital management of self-harm is an essential component of suicide prevention. Patients presenting to hospital for self-harm involving highly lethal methods or with high suicidal intent are a subgroup of self-harm patients at high risk of suicide. Investigating healthcare service provision from the patients' perspective is integral to the design and implementation of better quality care. The current study explored patients' experiences of engaging with healthcare services after a high risk self-harm presentation to hospital.

Methods A sequential transformative mixed-methods design was used. Quantitative information was obtained by interview administered questionnaires (including internationally validated scales, closed and open-ended questions) 0-3 months following a high risk self-harm presentation to a hospital emergency department $(n=67)$. Semi-structured follow-up interviews were conducted 6-9 months later providing qualitative data $(n=31)$. Follow-up interviews were recorded, transcribed and thematically analysed using NVivo software. Quantitative information was analysed in SPSS Version 25. Both methodologies were integrated during the interpretation of the results.

Results After the self-harm presentation, 85.7\% of patients reported receiving follow-up care with public outpatient mental health services and $61.8 \%$ attended their general 